\title{
Traumatismo obstétrico
}

\author{
Muñoz-Terrones MDE*, Rivera-Barragán V $^{* *}$, Terrones-Saldívar MC**
}
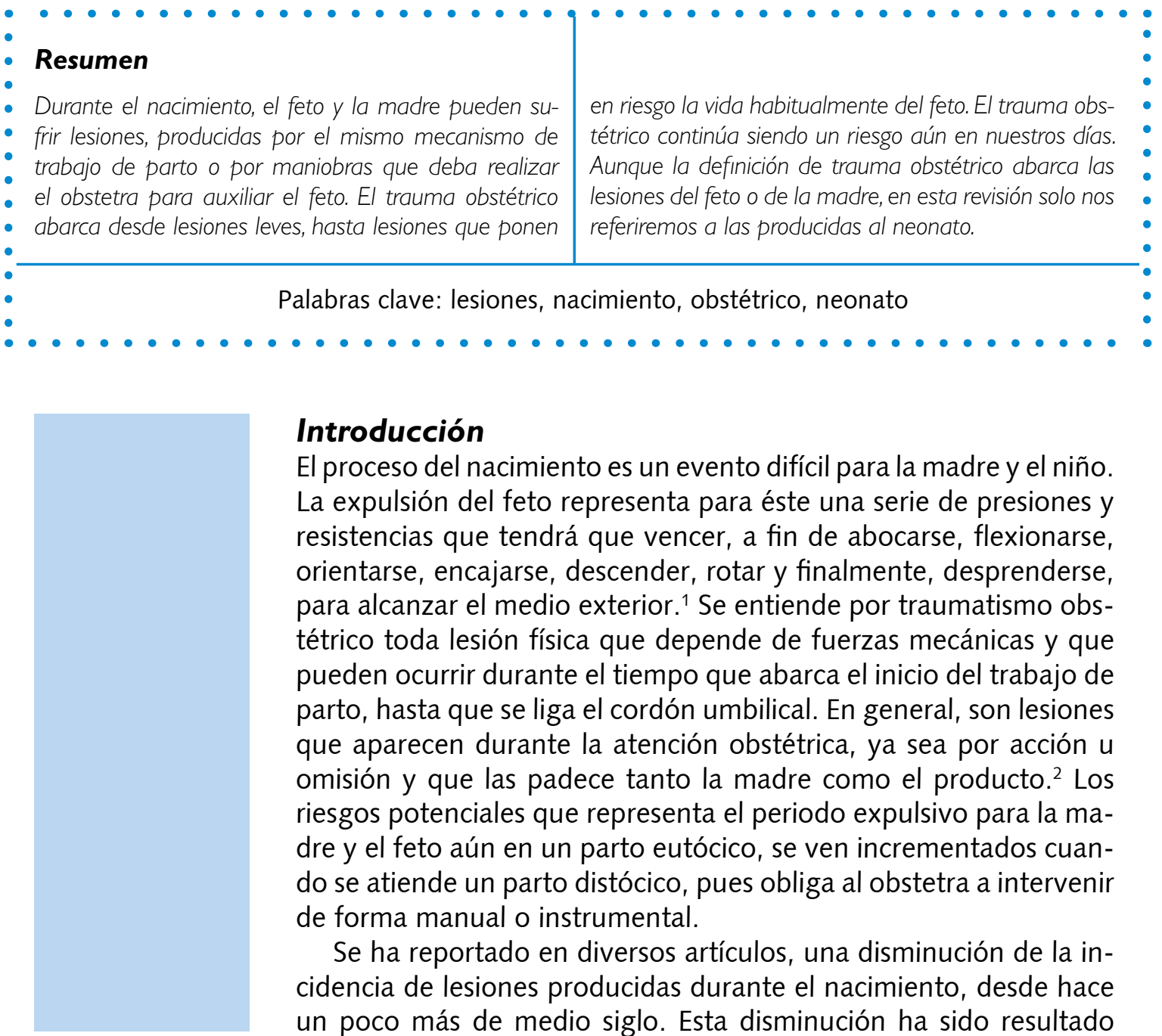

\section{Introducción}

El proceso del nacimiento es un evento difícil para la madre y el niño. La expulsión del feto representa para éste una serie de presiones y resistencias que tendrá que vencer, a fin de abocarse, flexionarse, orientarse, encajarse, descender, rotar y finalmente, desprenderse, para alcanzar el medio exterior. ${ }^{1}$ Se entiende por traumatismo obstétrico toda lesión física que depende de fuerzas mecánicas y que pueden ocurrir durante el tiempo que abarca el inicio del trabajo de parto, hasta que se liga el cordón umbilical. En general, son lesiones que aparecen durante la atención obstétrica, ya sea por acción u omisión y que las padece tanto la madre como el producto. ${ }^{2}$ Los riesgos potenciales que representa el periodo expulsivo para la madre y el feto aún en un parto eutócico, se ven incrementados cuando se atiende un parto distócico, pues obliga al obstetra a intervenir de forma manual o instrumental.

Se ha reportado en diversos artículos, una disminución de la incidencia de lesiones producidas durante el nacimiento, desde hace un poco más de medio siglo. Esta disminución ha sido resultado

* Estudiante del séptimo semestre de medicina, Centro de Ciencias de la Salud, UAA

** Profesores investigadores del Centro de Ciencias de la Salud.

Fecha de recibido 1 de diciembre 2010

Fecha de aceptación 15 de diciembre 2010

Correspondencia Dr Virgilio Rivera Barragán, Profesor investigador del Depto de Cirugía del Centro de Ciencias de la Salud, UAA. Av Universidad \#910 Ciudad Universitaria Ags., CP 20100 tel 9108436 Correo electrónico virgi_rivera@yahoo.com.mx 
de un mejor entendimiento del proceso del trabajo de parto, de la disponibilidad de tecnología que permite identificar las posibles situaciones de riesgo de trauma obstétrico, así como de la facilidad con que ahora se realizan las cesáreas para resolver riesgos perinatales como la macrosomía y la desproporción cefalopélvica. Sin embargo, es innegable que todavía queda mucho por hacer para disminuir las lesiones ocurridas al nacimiento, como por ejemplo, capacitar adecuadamente a los residentes de la especialidad en el uso de fórceps y realmente desterrar malas prácticas obstétricas como la maniobra de Kristeller. Por supuesto que es crucial la atención prenatal como estrategia de identificar y controlar otros riesgos perinatales. En México existen informes de traumatismos al nacimiento cuyas cifras oscilan entre $2.1 \%$ a $40.2 \% .3$ Este tipo de lesiones ocurren en $2 \%$ de los niños que fallecen al nacer y ocupan el octavo lugar como causa de muerte neonatal ${ }^{4}$. Entre los factores que se han asociado a la presentación de lesiones durante el nacimiento se encuentran: macrosomía, desproporción cefalopélvica, primiparidad, distocia de hombros, prematurez, trabajo de parto prolongado, presentación anormal (pélvica, occípito posterior o compuesta), alteraciones del cordón, sufrimiento fetal, malformaciones del neonato y aplicación de fórceps. ${ }^{4,5,6,7}$.

La cesárea ha traído consigo disminución de complicaciones en los fetos con presentaciones anómalas, como la pélvica, en los fetos macrosómicos, y en la desproporción cefalopélvica, lo que ha originado una reducción aproximadamente $90 \%$ en la frecuencia de lesiones originadas durante el nacimiento ${ }^{8}$.

De igual manera, el adecuado control prenatal, es importante para detectar a tiempo los factores de riesgo para este tipo de lesiones. Sin embargo el abuso de la opción quirúrgica para la interrupción del embarazo ha ocasionado otro tipo de lesiones como fracturas humerales, lesiones de la pared abdominal y heridas cortantes, entre otras. El uso de fórceps tiene un potencial efecto nocivo para el recién nacido principalmente en el cráneo y los nervios periférico. $^{2}$

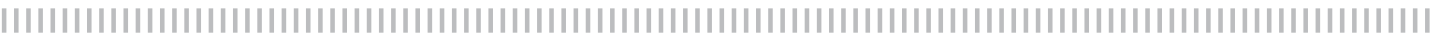

\section{Clasificación}

El trauma al nacimiento se divide en dos categorías de acuerdo a su etilología ${ }^{4}$ en que resulta de la hipoxia ó isquemia y las lesiones ocasionadas por las fuerzas mecánicas, originadas durante el trabajo de parto y el nacimiento. Estas últimas son las que se desarrollarán a continuación.

\section{Lesiones de Piel y Partes Blandas.}

\section{Necrosis de la grasa subcutánea}

Se trata de una lesión que se presenta en forma de placas o nódulos en el tronco, extremidades o cara, de tamaño variable a consecuencia de la presión continua o intensa sobre un área de tejido subcutáneo. Es indolora y a menudo sólo se aprecia a 
la palpación. No requiere tratamiento y remite a las pocas semanas después del nacimiento.

\section{Equimosis}

Constituyen las marcas más comunes como consecuencia de las fuerzas del parto sobre el producto. El interés en su reconocimiento, estriba en su diagnóstico diferencial con cuadros de coagulopatía o vasculitis. Son comunes en niños prematuros en presentación pélvica, ya que al nacer por parto vaginal o por cesárea son traccionados por las piernas. Pueden ser secundarios también a la aplicación de los fórceps.

\section{Laceraciones de la piel}

Pueden deberse al uso de fórceps en cuyo caso no hay ninguna indicación terapéutica. Ocasionalmente se han visto heridas limpias, efectuadas con el bisturí en el momento de abrir la cavidad uterina durante la cesárea, la mayoría de las lesiones se localizan en la cara u orejas, requieren sutura quirúrgica y se debe evitar su infección.

\section{Fracturas del cartílago nasal}

Estas fracturas ocurren cuando el fórceps se aplica sobre la nariz o se intenta una maniobra de Mariceaux en condiciones difíciles, la lesión más frecuente es la dislocación del septum de su sitio de inserción en el surco vomeriano y la columnela, el diagnóstico se hace cuando se observa desigualdad de las fosas nasales, debe realizarse el tratamiento de forma temprana puesto que puede conllevar dificultad respiratoria considerable.

\section{Hematoma del esternocleidomastoideo}

Es una lesión que resulta del estiramiento excesivo del cuello, cuando hay tracción de cabeza en fetos macrosómicos. La ruptura de las fibras musculares ocasiona el hematoma que se manifiesta por una tumoración de uno o dos centímetros en el trayecto del músculo, la detección temprana evita posibles complicaciones como la atrofia muscular con acortamiento de la longitud del músculo y por la tanto la aparición de tortícolis cuya presentación consiste en flexión del cuello ipsilateral a la lesión con rotación contralateral de la cabeza y barbilla ligeramente elevada, esta postura es fija y la cabeza no puede llevarse a una posición normal. El tratamiento consiste en estiramiento que deben realizarse varias veces al día. Se consiguen buenos resultados en más del $90 \%$ de los casos, si el diagnóstico y el inicio del tratamiento se llevan a cabo precozmente.

\section{Lesiones extracraneales}

\section{Caput succedaneum}

Consiste en una colección fluida serohemática entre la piel y el periostio. Lesión frecuente cuyas manifestaciones clínicas consisten en una tumefacción blanda de bordes irregulares que no tiene relación alguna con las suturas craneales. No requiere tratamiento y se resuelve espontáneamente en pocos días.

\section{Cefalohematoma}

Lesión hemorrágica extracraneal más frecuente, que puede ocurrir en $1-2 \%$ de los partos normales. Son lesiones comunes producidas por la ruptura de vasos subperiósticos que se manifiestan en forma de tumoración bien delimitada por las suturas craneales. En general se sitúan sobre los huesos parietales u occipitales. Su aparición está ligada al uso de maniobras expulsivas que involucran el uso de fórceps. Se asocia a fractura lineal del hueso subyacente en un $5 \%$ de los ca$\operatorname{sos}^{6,9,10}$. No requiere tratamiento, son raras las complicaciones como la infección, si aparece está indicado su drenaje.

\section{Hemorragia subgaleal}

Consiste en un sangrado entre la aponeurosis galeal del cuero cabelludo y el periostio. Se produce por la ruptura de alguna vena emisiaria que discurre entre los espacios 
subdural y subgaleal. Este último espacio queda limitado por las órbitas anteriormente, el occipucio posteriormente y los pabellones auriculares lateralmente. El volumen de sangre que puede acumularse en él es muy importante, en ocasiones pueden ocasionar shock hipovólemico por hemorragia masiva. Se considera que cada centímetro que aumente el perímetro cefálico se correlaciona con $38 \mathrm{ml}$ de sangre acumulada. Típicamente se detecta en las primeras horas de vida y puede crecer durante las próximas 72 hrs. El tratamiento es conservador, administrando paquetes globulares en caso necesario. Se le atribuye una mortalidad de hasta el $22 \%$ aunque para los supervivientes el pronóstico es excelente. ${ }^{6}$

\section{Lesiones craneales}

\section{Fracturas de los huesos del cráneo}

La mayoría son lineales y se presentan como consecuencia de una mala aplicación del fórceps o por la presión que ejercen las estructuras óseas maternas sobre el cráneo fetal. La complicación más frecuente es un quiste leptomeníngeo que puede separar los bordes de la lesión, también puede producir irritación cortical y desencadenar convulsiones. Esta complicación es indicación quirúrgica. Las fracturas deprimidas producidas por traumatismo del parto pueden llegar a suponer el $16 \%$ de las fracturas craneales en estos neonatos. El 75\% se producen en la región parietal y el 15\% son frontales. Las indicaciones de cirugía reconstructiva son: a) fragmento óseo libre en parénquima cerebral, b) déficit neurológico, c) aumento de la presión intracraneal y d) fístula de LCR. ${ }^{11}$

\section{Lesiones intracraneales}

La prevalencia de estas lesiones en su forma sintomática se sitúa entre 5-6 casos por cada 10,000 recién nacidos vivos. ${ }^{9}$ Clínicamente suelen manifestarse dentro de las primeras 48 horas de vida, mediante convulsiones, alteraciones del estado de con- ciencia, focalizaciones o apneas.

Las hemorragias intracraneales pueden ser epidural, subdural, subaracnoidea, intraventricular o intraparenquimatosa. Los hematomas subdurales pueden producirse por ruptura de venas corticales o lesión de senos venosos, especialmente en las desproporciones cefalopélvicas, prematuros, prímipara o multípara añosa con parto prolongado, o en presentaciones anormales que requiere el uso de fórceps. Clínicamente se observa rigidez de nuca, bradicardia, anisocoria, hemiparesia, hipotensión y alteraciones de cardiorespiratorias. El diagnóstico se hace mediante TAC o RM. El tratamiento es la evacuación del hematoma mediante trépanos o craneotomía.

El hematoma epidural supone el $2 \%$ de las hemorragias cerebrales neonatales ${ }^{11}$. La mayoría debido a ruptura de vasos meníngeos, ocurren en partos prolongados $\mathrm{y} / \mathrm{o}$ asistidos por fórceps. Los pacientes muestran fontanela tensa, déficits neurológicos focales e hipotensión. El diagnóstico también se realiza por TAC o RM. El tratamiento consiste en drenaje.

Las hemorragias subaracnoidea e intraparenquimatosa son raras, siendo más habituales en prematuros de peso inferior a 2,000 g o menores de 30 semanas de gestación, por cambios hipóxicos o isquémicos que puedan añadirse a un parto traumático.

La hemorragia intraventricular es la variante más común de las hemorragias intracraneales en el neonato ${ }^{12}$. Se presenta sobre todo en menores de 32 semanas de edad gestacional; su frecuencia oscila entre 40 y $50 \%^{13}$. Aproximadamente el $80 \%$ se rompe hacia el ventrículo y se acumulan en la fosa posterior; la lesión básica es el sangrado de la matriz germinal subependimaria ${ }^{12}$. El cuadro clínico puede ser súbito o de evolución lenta, el primero cursa con alteraciones del estado de conciencia pasando de estupor a coma en cuestión de horas, existe apnea, crisis convulsivas tónicas generalizadas, actitud de descere- 
bración, arreflexia pupilar y cuadriparesia flácida. En la forma lenta la evolución es de horas o días y presenta estupor, hipotonía y desviación de los globos oculares. El pronóstico es malo para ambas formas de presentación, en el cuadro agudo la muerte es súbita y en el caso de una evolución tardía existen secuelas neurológicas que dependen de la localización y extensión de la lesión ${ }^{13}$.

\section{Lesiones Neurológicas}

\section{Parálisis Facial}

El nervio facial es el par craneal que se ve afectado con mayor frecuencia en caso de traumatismo obstétrico, dado su recorrido superficial sobre la mastoides y la mandíbula, lo que lo hace susceptible a compresiones durante el parto por los huesos pélvicos, o más frecuentemente por el uso de fórceps. Aparece en 0.6 de cada 1000 recién nacidos vivos $^{9}$. El cuadro clínico es un neonato que presenta la piel lisa en la hemicara ipsilateral a la lesión, el ángulo labial caído, la frente sin arrugas, y el ojo abierto, la parálisis es flácida y aparece desde el nacimiento y se hace más evidente cuando el niño llora.

La parálisis facial suele asociarse con otros tipos de traumas durante el proceso del parto, y ocurren con mayor frecuencia en fetos macrosómicos obtenidos por vía vaginal, hijos de primigestas y uso de fórceps. El tratamiento consiste en "esperar y ver" la mayoría de los casos se resuelven a las 24 hrs de haber iniciado, o en los primeros días, mientras tanto, se protege la córnea con un lubricante artificial y se cubre el ojo con un apósito. En estos casos el pronóstico es bueno con recuperación funcional completa. Si no hay mejoría a los 10 días debe comenzarse el tratamiento con fisioterapia. La cirugía sólo está indicada cuando se comprueba la disrupción de las fibras nerviosas.

\section{Lesión del Plexo Braquial}

Su frecuencia es variable de un país a otro, la literatura indica, 1 cada 6000 partos, otros 1 por 1500 partos, es más común en parto en podálica ${ }^{14}$.

Se trata de una lesión local que afecta exclusivamente al miembro superior. Ocurre con más frecuencia en partos inducidos e instrumentados que en espontáneos, ya que muchas veces requieren maniobras, especialmente en pelvis estrechas o planas no diagnosticadas, o en casos de fetos macrosómicos. Se asocia con cierta frecuencia a las fracturas de clavícula y húmero, a tortícolis muscular congénita y a parálisis facial. El miembro superior derecho es el más afectado, producto de ser más frecuente la presentación occípito ilíaca izquierda anterior. La lateralidad de la lesión depende de la rotación de la cabeza a través del canal del parto.

La patogénesis de la lesión implica generalmente la tracción del plexo nervioso durante la extracción de los hombros. Existen tres tipos de lesión del plexo braquial, según las raíces nerviosas afectadas.

- Parálisis braquial superior o de Erb.: las raíces lesionadas son C5- C7, representa el $90 \%$ de los casos. Se observa la extremidad afectada en adducción, rotación interna del hombro, extensión del codo, pronación del antebrazo y flexión de la muñeca y de los dedos, a la exploración física el reflejo bicipital está ausente. En ocasiones se acompaña de parálisis frénica ipsilateral.

- Parálisis braquial inferior o de Klumpke: Se afectan las raíces C8 y T1, ocurre en menos del $1 \%$ de los casos. Existe parálisis de la mano, ausencia del reflejo de prensión, extensión del codo y conservación de los reflejos profundos. Puede asociarse a síndrome de Horner homolateral y presentar conjuntamente ptosis, miosis y endoftalmos.

- Parálisis braquial completa o total. Todas las raíces del plexo braquial se ven afectadas, lo que origina una parálisis flácida de toda la extremidad superior. Representa el $10 \%$ de todos los casos. 
El diagnóstico es eminentemente clínico, y la evolución durante los primeros días nos permite establecer el pronóstico. Una recuperación completa será posible si la flexión del codo y la abducción del hombro se recuperan antes de los dos meses de edad, lo cual ocurre en el $90 \%$ de los casos, siendo principalmente en las lesiones altas (C5 y C7). Se estima que sólo entre $5.5 \%$ y $6.7 \%$ evolucionará a una lesión neurológica irreversible ${ }^{15}$. Síndrome de Horner ipsilateral, fracturas asociadas, lesión del nervio frénico, ensombrecen el pronóstico ${ }^{14}$. El tratamiento consiste en colocar el brazo a $90^{\circ}$ con respecto al tronco, antebrazo a $90^{\circ}$ con relación al brazo; se fija esta posición durante siete a diez días al cabo de los cuales se inician movimientos pasivos suaves varias veces al día. Es necesario iniciar fisioterapia lo más pronto posible. Si no se aprecia ningún tipo de mejoría más allá de los seis meses se debe considerar el tratamiento quirúrgico.

\section{Lesiones del nervio frénico}

Tracción de las raíces cervicales C3, C4, y C5, conlleva parálisis del diafragma ipsilateral a la lesión, en el $75 \%$ de los casos se asocia a parálisis braquial.

Suele ser unilateral y de predominio derecho. La clínica consiste en distrés respiratorio, con menor movilización del hemitórax afectado. La radiografía de tórax confirma el diagnóstico. El tratamiento consiste en soporte respiratorio y en más del $75 \%$ de los pacientes es necesario el tratamiento quirúrgico, mediante plicatura diafragmática.

\section{Lesión de la médula espinal}

El trauma medular se produce cuando se tracciona con fuerza a un niño que nace en presentación pélvica y presenta una retención de la cabeza. Representa 0.14\% de cada 10,000 neonatos vivos por lo que constituye una lesión rara ${ }^{12}$.

El diagnóstico se sospecha al sentirse un chasquido en el cuello al efectuar la tracción, el cual es resultado de la distensión ligamentosa y la separación de los cuerpos vertebrales, la médula que es blanda y de longitud definida, no tolera la elongación y puede desgarrarse. Las manifestaciones clínicas son hipotermia, hipotonía, disnea, alteraciones del estado de conciencia, que conlleva la muerte si la sección ha sido completa. En otros casos desarrollan manifestaciones clínicas tardías: distrés respiratorio, flaccidez muscular, paresia de extremidades, arreflexia y compromiso de esfínteres. Las lesiones pueden ser cervicales o tóraco-lumbares siendo su frecuencia aproximadamente la misma. El pronóstico es malo conlleva secuelas graves e incapacitantes: paresias, tetraparesias, escoliosis, trastornos del aprendizaje o del comportamiento y crisis convulsivas. Los varones son más susceptibles a desarrollar trauma medular. El tratamiento está dirigido a mantener el estado general en la mejor forma posible, a evitar infecciones recurrentes y a efectuar terapia física.

\section{Lesión del nervio laríngeo}

Se presenta principalmente en el lado izquierdo, se manifiesta en forma de estridor, distrés respiratorio, Ilanto disfónico, dificultades para la deglución y riesgo de aspiración como consecuencia de la parálisis de la cuerda vocal ipsilateral. Suele resolverse espontáneamente en las primeras 24 horas. Cuando es bilateral empeora el pronóstico.

\section{Lesiones óseas}

\section{Fractura de clavícula}

La clavícula es el hueso que más frecuentemente se fractura durante el parto. Ocurre más frecuentemente en distocia de hombros en productos macrosómicos. Es la consecuencia de maniobras de tracción bruscas sobre el feto ${ }^{16}$. En presentación podálica la extensión de los brazos sobre la cabeza ejerce una exagerada presión sobre el hueso, la cual se acentúa más al pasar por el estrecho pelviano inferior. La mayoría de las fracturas son en tallo ver- 
de, pero también pueden ser completas, se aprecia una asimetría del reflejo de Moro, en ocasiones con crepitación sobre la zona de la fractura. El diagnóstico se confirma radiológicamente. El tratamiento consiste en inmovilización de la extremidad superior con un cabestrillo para el control del dolor, durante dos a tres semanas.

\section{Fractura del húmero}

Es más frecuente en la atención de un parto pélvico, las formas incompletas pueden pasar desapercibidas y ser un hallazgo radiológico, o palparse el callo óseo durante un examen de rutina. En las fracturas completas suele existir desplazamiento de los cabos óseos y deformidad de la extremidad, el neonato se halla en posición antiálgica, elimina el reflejo de Moro y manifiesta edema local muy evidente. El tratamiento consiste en inmovilización del brazo sobre el tórax con un vendaje durante dos a tres semanas ${ }^{10}$.

\section{Fractura del fémur}

El sitio de fractura, por lo general, es el tercio superior donde el hueso es más delgado, con mucha frecuencia la fractura es completa. Existe edema local, deformación e inmovilidad del muslo afectado. El tratamiento es la inmovilización de la extremidad por tres semanas. Se considera una lesión poco frecuente $e^{6,10,12}$.

\section{Ruptura viscerales}

\section{Hemorragia hepática}

Es poco común y puede pasar desapercibida inicialmente y manifestarse luego de manera tan brusca que no da tiempo de iniciar medidas terapéuticas. La hemorragia es usualmente subcapsular, se origina en la ruptura de vasos que se traumatizan al traccionar por el abdomen a un niño que ha nacido en presentación podálica. Los signos clínicos pueden estar ausentes durante la primera semana de vida mientras el hematoma crece, al romperse viene un shock hipovólemico. El incremento de la palidez y la defensa abdominal hacen sospechar el diagnóstico. El tratamiento siempre es quirúrgico, con reposición de las pérdidas sanguíneas ${ }^{6,10}$.

\section{Ruptura del bazo}

Son menos frecuentes aún que la ruptura hepática. Cursa con un cuadro de shock hipovólemico y es necesaria la exploración quirúrgica para detectar el sitio de sangrado. El uso de fibrina y la celulosa ha permitido efectuar más fácilmente la reparación de las laceraciones y evitar la esplenectomía ${ }^{9,10}$.

\section{Hemorragia adrenal}

La etiología no es clara y se cree que es multifactorial. Las manifestaciones clínicas varían de acuerdo a la intensidad de la hemorragia, el paciente manifiesta fiebre, taquicardia, palidez y cianosis, existe además anorexia, diarrea, deshidratación, uremia, hipoglicemia, y vómito. El cuadro suele ser de difícil control, los pacientes en estado grave presentan choque y mueren en pocas horas. El tratamiento depende del grado de hemorragia y de acuerdo con la extensión del proceso parenquimatoso, está indicada la adrenalectomía9,10,12.

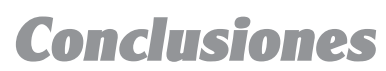

En la actualidad, el conocimiento de la fisiología fetal, así como de los procesos técnicos encaminados a la vigilancia de la estabilidad del producto durante la gestación y, sobre todo, durante el proceso del parto, aunado al perfeccionamiento en las técnicas obstétricas, han incrementado el margen de seguridad para el neonato y por tanto en buenas condiciones de asistencia médica con personal calificado las 
lesiones por traumatismo fetal son cada vez menores.

Las lesiones que se producen en los neonatos tienen un origen multifactorial en donde intervienen factores maternos, neonatales y de la atención del parto. Está demostrado que los fetos macrósomicos tienen mayor riesgo de presentar complicaciones al momento del parto.

El traumatismo al nacimiento se ha convertido en un indicador de la calidad de la atención obstétrica de las instituciones de atención materno-infantil. Por lo que la supervisión y enseñanza tutelar del personal médico en formación parece ser la clave para disminuir el número y gravedad las lesiones.

Es necesario tener en cuenta que el trauma obstétrico constituye un grupo heterogéneo que puede abarcar desde lesiones leves hasta lesiones que pueden comprometer la vida del neonato en cuestión de minutos. En consecuencia el diagnóstico temprano y tratamiento oportuno permiten disminuir la mortalidad neonatal asociada al parto y las secuelas a largo plazo. La indicación quirúrgica en los recién nacidos depende del balance riesgo-beneficio de la cirugía.

La aplicación de maniobras obstétricas durante el parto es todavía frecuente en nuestro medio, sin embargo el índice de complicaciones que provocan suele ser mayor al beneficio reportado, la indicación precisa y la realización correcta de la técnica podrían disminuir la incidencia de trauma obstétrico.

Finalmente un buen control prenatal y la identificación de madres con factores de riesgo, así como de productos macrosómicos permitirían un mejor control de las variables impredecibles durante el nacimiento, permitiendo una adecuada planeación por lo que en estos casos estaría indicada la realización de la extracción del producto mediante cesárea.

\section{Bibliografía}

1 Caldera Rodarte S. Trauma obstétrico. Gineco 2007; 14 (88): 6-23.

2 Baños Toscano L., Incidencia y factores de riesgo en trauma obstétrico en recién nacidos en el hospital "Ignacio Zaragoza". Revista de especialidades médico - quirúrgicas, 2007;12(2): 32-36.

3 Delgado Becerra A. Características del traumatismo al nacimiento en una institución del tercer nivel de atención., Revista de Perinatología y Reproducción Humana 2003; 17: 169-175.

4 Ponce de León Tapia M.M., Hernández Sánchez M.D., Ibarra Vela R.A., Rosas Sotelo S., Valdivia Ballon A., Frecuencia y tipo de lesiones obstétricas en neonatos nacidos en un hospital general., Revista Mexicana de Pediatría 2000; 67 (4): 161-165.

5 Posada Arévalo S.E., Zavala González M.A., Prevalencia de macrosomía fetal en Cárdenas, Tabasco., Arch Invest Pediátr Méx 2007; 10(2):5-10.

6 Botero J; Jubiz A; Henao G; Obstetricia y Ginecología; $7^{a}$ edición. Organización Panamericana de salud. Colombia, 2004, Pp 359-365.

7 Molina Hernández O.R., Monteagudo Ruiz C.L., Caracterización perinatal del recién nacido macrosómico., Revista Cubana de Obstetricia y Ginecología 2010; 36 (3): 313-321.

8 García H., Rubio Espíritu J., Islas Rodríguez M.T., Factores de riesgo asociados a traumatismo al nacimiento., Revista de investigación clínica 2006; 58(5): 416-423.

9 Cabero L; Saldívar D; Cabrilo E; Obstetricia y Medicina Materno-Fetal. Editorial Médica- Panamericana., México, D.F. 2007.
Pp 1169-1173.

10 Cunningham F.G; Levero K; Bloom S. et al. Obstetricia de Williams. 22da Edición. Editorial Mc-Graw Hill Interamericana. México, D.F. 2005. Pp 681-685.

11 Villarejo F., Belinchón J.M., Carceller F., Gómez Sierra A., Pas cual A.,Cordobés F., Pérez- Díaz C., Rivero B., Lesiones craneales secundarias a parto asistido con fórceps., Neurología 2009; 20: 262-264.

12 Morales Bastos C., Lesiones hipoxico isquémicas del sistema nervioso central en el período perinatal., Revista Española de Patología 2002; 35(1): 5-20.

13 Ávalos Chavez L.M., Lesiones al nacimiento. Tomado de: http://www.pediatriaenlinea.com/pdf/lesionalnacimiento.pdf $31 / 11 / 10$

14 Lattus Olmos J., Pesse B.D., Trauma obstétrico parálisis braquial obstétrica., Rev. Obstet. Ginecol. 2009; 4(1): 66-77.

15 Albornoz V.J.,Salinas P.H., Reyes P.A., Morbilidad fetal asociada al parto en macrosómicos: análisis de 3981 nacimientos., Rev. Chil Obstet Ginecol 2005; 70(4): 218-224.

16 Pérez R., Andaluz P., Arriagada M.,Oyarzún C.,Urrutia P., Fractura de clavícula en recién nacidos: factores de riesgo y morbilidad asociada., Prog Obstet Ginecol. 2006; 49(3): 121-6. 